Pacific

Journal of

Mathematics

\title{
ON THE EXISTENCE OF CENTRAL FANS OF CAPILLARY SURFACES
}

AMMAR KHANFER

Volume $281 \quad$ No. 2

April 2016 


\title{
ON THE EXISTENCE OF CENTRAL FANS OF CAPILLARY SURFACES
}

\author{
AMMAR KHANFER
}

\begin{abstract}
We prove that under some conditions every nonparametric capillary surface which has a central fan (of radial limits at a point $\mathcal{O}$ ) can be perturbed with respect to the contact angle and the perturbed surfaces continue to have central fans. In particular, any nonparametric capillary surface which is symmetric with respect to a vertical plane through $\mathcal{O}$ and has a central fan may be perturbed (with respect to the contact angle) in a nonsymmetric manner and the resulting capillary surfaces will not be symmetric with respect to the vertical plane but will continue to have central fans.
\end{abstract}

\section{Introduction}

Let $\Omega$ be a bounded open set in $\mathbb{R}^{2}$ with locally Lipschitz boundary $\partial \Omega$ such that a point $\mathcal{O}$ lies on $\partial \Omega, \partial \Omega \backslash\{\mathcal{O}\}$ is a $C^{2}$ curve and there exist distinct rays $l^{ \pm}$starting at $\mathcal{O}$ such that $\partial \Omega$ is tangent to $l^{+} \cup l^{-}$at $\mathcal{O}$. By rotating and translating the domain, we may assume $\mathcal{O}=(0,0), l^{+}=\{r(\cos \alpha, \sin \alpha): r \geq 0\}, l^{-}=\{r(\cos \alpha,-\sin \alpha): r \geq 0\}$ and

$$
\Omega \cap B(\mathcal{O}, \delta)=\left\{r(\cos \theta, \sin \theta): 0<r<\delta, \theta^{-}(r)<\theta<\theta^{+}(r)\right\}
$$

for some $\alpha \in(0, \pi), \delta>0$ and functions $\theta^{ \pm} \in C^{0}([0, \delta))$ which satisfy $\theta^{-}<\theta^{+}$, $\theta^{-}(0)=-\alpha$ and $\theta^{+}(0)=\alpha$; here $B(\mathcal{O}, \delta)$ is the open ball in $\mathbb{R}^{2}$ centered at $\mathcal{O}$ of radius $\delta$. We will assume this description of $\Omega$ holds throughout this paper.

Let $\gamma$ be a measurable function mapping $\partial \Omega$ into $[0, \pi]$ and $f \in C^{2}(\Omega) \cap L^{\infty}(\Omega)$ be a (bounded) variational solution of the nonparametric capillary surface problem of finding a function $u \in C^{2}(\Omega)$ such that

$$
\begin{array}{ll}
\operatorname{div}(T u)=\kappa u+\lambda & \text { in } \Omega, \\
T u \cdot v=\cos \gamma & \text { a.e. on } \partial \Omega,
\end{array}
$$

where

$$
T u=\left(\frac{D_{1} u}{\sqrt{1+|D u|^{2}}}, \frac{D_{2} u}{\sqrt{1+|D u|^{2}}}\right)
$$

MSC2010: primary 35J93, 76B45; secondary 35J62, 53A10.

Keywords: capillary surfaces, nonconvex corner, central fans. 
$\kappa$ and $\lambda$ are constants and $\nu$ is the outer unit normal to $\partial \Omega$. We will assume $\kappa>0$ and therefore, by vertical translation, assume $\lambda=0$. (Since $\kappa>0, f$ is unique.)

Lancaster and Siegel [1996] proved that if $\gamma$ is bounded away from 0 and $\pi$ near $\mathcal{O}$, then the radial limit of $f$ at $\mathcal{O}$ in the direction $\theta$,

$$
R f(\theta) \stackrel{\text { def }}{=} \lim _{r \downarrow 0} f(r \cos \theta, r \sin \theta),
$$

exists for each $\theta \in[-\alpha, \alpha], R f$ belongs to $C^{0}([-\alpha, \alpha]), R f(-\alpha)$ is the limiting height at $\mathcal{O}$ of the trace of $f$ on $\partial^{-} \Omega=\partial \Omega \cap\{y<0\}$ and $R f(\alpha)$ is the limiting height at $\mathcal{O}$ of the trace of $f$ on $\partial^{+} \Omega=\partial \Omega \cap\{y>0\}$. In particular, when $\alpha>\frac{\pi}{2}$, so that $\partial \Omega$ has a nonconvex (or reentrant) corner at $\mathcal{O}$, and $f$ is discontinuous at $\mathcal{O}$, the conclusion of Theorem 1 of [Lancaster and Siegel 1996] is that the radial limits of $f$ behave in one of the following ways:

(i) There exist $\alpha_{1}$ and $\alpha_{2}$ so that $-\alpha \leq \alpha_{1}<\alpha_{2} \leq \alpha$ and $R f$ is constant on [ $\left.-\alpha, \alpha_{1}\right]$ and $\left[\alpha_{2}, \alpha\right]$ and is strictly increasing or strictly decreasing on $\left[\alpha_{1}, \alpha_{2}\right]$. Label these case (I) and case (D), respectively.

(ii) There exist $\alpha_{1}, \alpha_{L}, \alpha_{R}, \alpha_{2}$ so that $-\alpha \leq \alpha_{1}<\alpha_{L}<\alpha_{R}<\alpha_{2} \leq \alpha, \alpha_{R}=\alpha_{L}+\pi$, and $R f$ is constant on $\left[-\alpha, \alpha_{1}\right],\left[\alpha_{L}, \alpha_{R}\right]$, and $\left[\alpha_{2}, \alpha\right]$ and is either strictly increasing on $\left[\alpha_{1}, \alpha_{L}\right]$ and strictly decreasing on $\left[\alpha_{R}, \alpha_{2}\right]$ or strictly decreasing on $\left[\alpha_{1}, \alpha_{L}\right]$ and strictly increasing on $\left[\alpha_{R}, \alpha_{2}\right]$. Label these case (ID) and case (DI), respectively.

In addition, if the limits

$$
\gamma_{1}=\lim _{\partial^{+} \Omega \ni(x, y) \rightarrow \mathcal{O}} \gamma(x, y) \text { and } \gamma_{2}=\lim _{\partial^{-} \Omega \ni(x, y) \rightarrow \mathcal{O}} \gamma(x, y)
$$

both exist, then [Lancaster 2010; 2012; Lancaster and Siegel 1996] imply that $\alpha_{2}$ equals $\alpha-\gamma_{1}$ in cases (I) and (DI) and $\alpha+\gamma_{1}-\pi$ in cases (D) and (ID) while $\alpha_{1}$ equals $-\alpha+\gamma_{2}$ in cases (D) and (DI) and $\pi-\alpha-\gamma_{2}$ in cases (I) and (ID).

The intervals in $[-\alpha, \alpha]$ on which $R f$ is constant are called "fans" in, for example, [Lancaster 1985]; specifically, $\left[-\alpha, \alpha_{1}\right]$ and $\left[\alpha_{2}, \alpha\right]$ are called "side fans" and, if it exists, $\left[\alpha_{L}, \alpha_{L}+\pi\right]$ is called a "central fan". When $\Omega$ and $\gamma$ are symmetric with respect to the $x$-axis, we have $R f(\alpha)=R f(-\alpha)$ and, if $\alpha>\frac{\pi}{2}, \alpha_{L}=-\frac{\pi}{2}$ and $\alpha_{R}=\frac{\pi}{2}$. (If $\kappa<0$ in (1), we would need to explicitly assume $f(x, y)=f(x,-y)$ for $(x, y) \in \Omega$.) If the fans touch or overlap (e.g., $\gamma_{1}+\gamma_{2} \geq 2 \alpha-\pi$ in a situation where case (DI) would hold), then $f$ is continuous at $\mathcal{O}$.

Let $\Omega$ be a bounded domain in $\mathbb{R}^{2}$ which is symmetric with respect to the $x$-axis and has a reentrant corner of size $2 \alpha>\pi$ at the origin $\mathcal{O}$. Let $\gamma: \partial \Omega \rightarrow(0, \pi)$ also be symmetric with respect to the $x$-axis such that the limits in (3) exist and $\gamma_{1}=\gamma_{2}<\frac{\pi}{2}$. As in Example 2 of [Lancaster and Siegel 1996], it follows that the solution $f$ of (1)-(2) with the domain $\Omega$ and contact angle $\gamma$ above is continuous 
at $\mathcal{O}$ if and only if $\gamma_{1} \geq \frac{\pi}{2}-\alpha$ and the radial limits $R f(\theta)$ of $f$ at $\mathcal{O}$ have a central fan if $\gamma_{1}<\frac{\pi}{2}-\alpha$. Danzhu Shi and Robert Finn [2004] considered the borderline case in which $\gamma_{1}=\alpha-\frac{\pi}{2}$, so that $f$ is continuous at $\mathcal{O}$. By perturbing the domain (using "an asymmetric domain perturbation that is in an asymptotic sense arbitrarily small"), they showed that the solution of the perturbed capillary problem is discontinuous at $\mathcal{O}$. (They convert the behavior of the radial limit function from a constant in Example 2 to case (I) in the perturbed problem.)

Consider a similar (symmetric) situation with a constant contact angle $\gamma$ which satisfies $\gamma<\alpha-\frac{\pi}{2}$, so that the solution $f$ of (1)-(2) with the (symmetric) domain $\Omega$ and contact angle $\gamma$ is discontinuous at $\mathcal{O}$, the radial limits $R f(\theta)$ of $f$ at $\mathcal{O}$ have a central fan and case (DI) holds. Applying the procedure of Finn and Shi, one makes a suitable, nonsymmetric (with respect to the $x$-axis) perturbation of $\Omega$ outside a neighborhood of $\mathcal{O}$ and obtains a new solution $\tilde{f}$ of (1)-(2) in the perturbed domain $\widetilde{\Omega}$, and one then shows that $\tilde{f}$ is discontinuous at $\mathcal{O}$ and the radial limits $R \tilde{f}(\theta)$ have no central fan (i.e., case (I) holds); the size of the domain perturbation required to achieve this depends on the size of $\alpha-\frac{\pi}{2}-\gamma$.

We might view their example and procedure as a perturbation of the contact angle in a fixed domain $\widehat{\Omega}$ as follows. Let $\widehat{\Omega}$ be the largest open subset of $\Omega \cap \widetilde{\Omega}$ which is symmetric with respect to the $x$-axis. Let $\hat{v}$ denote the exterior unit normal to $\widehat{\Omega}$ at points of $\partial \widehat{\Omega}$ where it exists. Define (variable) contact angles $\lambda, \tilde{\lambda}: \partial \widehat{\Omega} \rightarrow[0, \pi]$ as follows:

- On $\partial \widehat{\Omega} \cap \partial \Omega$, set $\lambda=\gamma$.

- On $\partial \widehat{\Omega} \cap \partial \widetilde{\Omega}$, set $\tilde{\lambda}=\gamma$.

- On $\partial \widehat{\Omega} \cap \Omega$, set $\lambda=T f \cdot \hat{v}$ when $\hat{v}$ is defined; recall that $f \in C^{2}(\Omega)$.

- On $\partial \widehat{\Omega} \cap \widetilde{\Omega}$, set $\tilde{\lambda}=T \tilde{f} \cdot \hat{v}$ when $\hat{v}$ is defined; recall that $\tilde{f} \in C^{2}(\widetilde{\Omega})$.

Using the procedure given in [Shi and Finn 2004], notice that $\hat{v}$ exists at all but a finite number of points and so $\lambda$ and $\tilde{\lambda}$ are defined almost everywhere on $\partial \widehat{\Omega}$. From Theorem 5.1 of [Finn 1986], we see that $f$ and $\tilde{f}$ are the solutions of (1)-(2) with domain $\widehat{\Omega}$ and contact angles $\lambda$ and $\tilde{\lambda}$ respectively. We may therefore view $\tilde{\lambda}$ as a perturbation of the (symmetric) contact angle $\lambda$ and, when $\gamma<\alpha-\frac{\pi}{2}$, this perturbation $\tilde{\lambda}$ destroys the central fan. In this paper, we establish the stability of central fans with respect to sufficiently small perturbations of the contact angle $\gamma$, leaving the domain $\Omega$ fixed; this implies that $\tilde{\lambda}$ is a "large" perturbation of $\lambda$. We shall prove the following result.

Theorem 1. Suppose $\Omega$ is a bounded open domain in $\mathbb{R}^{2}$ which has a reentrant corner at $\mathcal{O}$ of size $2 \alpha$ with $\alpha \in\left(\frac{\pi}{2}, \pi\right)$ as described above. Suppose also that there is a finite set $A=\left\{P_{1}, \ldots, P_{m}\right\} \subset \partial \Omega$ with $m \geq 1$ and $P_{1}=\mathcal{O}$ such that $\partial \Omega \backslash A$ is a $C^{4}$ curve (if $m=1$ ) or a finite disjoint union of $C^{4}$ curves (if $\left.m>1\right)$. Let 
$\gamma \in C^{1, \beta}(\partial \Omega \backslash A)$, for some $\beta \in(0,1)$, satisfy $\delta_{0} \leq \gamma \leq \pi-\delta_{0}$ for some $\delta_{0}>0$ such that the limits

$$
\gamma_{1}=\lim _{\partial^{+} \Omega \ni(x, y) \rightarrow \mathcal{O}} \gamma(x, y) \text { and } \gamma_{2}=\lim _{\partial^{-} \Omega \ni(x, y) \rightarrow \mathcal{O}} \gamma(x, y)
$$

both exist. Suppose there exists $f \in C^{2}(\Omega) \cap L^{\infty}(\Omega)$ which satisfies (1)-(2) and is discontinuous at $\mathcal{O}$ and the radial limit function of $f$ at $\mathcal{O}, R f(\cdot)$, behaves as in case (ID) or case (DI).

Then there exist functions $\omega^{ \pm}: \partial \Omega \rightarrow[0, \pi]$ with $0 \leq \omega^{+} \leq \gamma \leq \omega^{-} \leq \pi$ on $\partial \Omega$ and $\omega^{+}<\gamma<\omega^{-}$on $\partial \Omega \backslash$ A such that if $\sigma: \partial \Omega \rightarrow(0, \pi)$ with $\omega^{+} \leq \sigma \leq \omega^{-}$a.e. on $\partial \Omega$ and $\delta_{1} \leq \sigma \leq \pi-\delta_{1}$ for some $\delta_{1} \in\left(0, \delta_{0}\right)$, then the radial limit function $R h$ of the solution $h \in C^{2}(\Omega)$ of (1)-(2) with $\gamma$ replaced by $\sigma$ in (2) has the same type of behavior (i.e., case (ID) or case (DI) holds) as does $R f$. In particular, the radial limits of h have a central fan.

The following corollary shows that Example 2 of [Lancaster and Siegel 1996] can be perturbed (with respect to the contact angle) and that the resulting nonsymmetric nonparametric capillary surfaces will have central fans.

Corollary 2. Let $\Omega$ be an open, connected, bounded Lipschitz domain which is symmetric with respect to the $x$-axis such that $\mathcal{O}=(0,0) \in \partial \Omega, \partial \Omega \backslash\{\mathcal{O}\}$ is a $C^{4}$ curve and $\Omega$ has a corner at $\mathcal{O}$ with opening angle $2 \alpha>\pi$. Suppose $\gamma: \partial \Omega \backslash\{\mathcal{O}\} \rightarrow$ $(0, \pi)$ is a $C^{1, \beta}$ map which satisfies $\gamma(x,-y)=\gamma(x, y)$ for $(x, y) \in \partial \Omega$ and for which the limit

$$
\lim _{\partial \Omega \ni(x, y) \rightarrow \mathcal{O}} \gamma(x, y)=\gamma_{0},
$$

exists and $0<\gamma_{0}<\alpha-\frac{\pi}{2}$. Let $f \in C^{2}(\Omega) \cap C^{1, \beta}(\bar{\Omega} \backslash\{\mathcal{O}\})$ of $(1)-(2)$. Then $f$ is discontinuous at $\mathcal{O}$, the radial limit function $R f$ behaves as in case $(D I)$ and there exist functions $\omega^{ \pm}: \partial \Omega \rightarrow[0, \pi]$ with $0 \leq \omega^{+} \leq \gamma \leq \omega^{-} \leq \pi$ on $\partial \Omega$ and $\omega^{+}<\gamma<\omega^{-}$on $\partial \Omega \backslash A$ such that if $\sigma: \partial \Omega \rightarrow(0, \pi)$ with $\omega^{+} \leq \sigma \leq \omega^{-}$a.e. on $\partial \Omega$ and $\delta_{1} \leq \sigma \leq \pi-\delta_{1}$ for some $\delta_{1} \in\left(0, \delta_{0}\right)$, then the radial limit function $R h$ of the solution $h \in C^{2}(\Omega)$ of (1)-(2) with $\gamma$ replaced by $\sigma$ in (2) is discontinuous at $\mathcal{O}$ and behaves as in case $(D I)$.

We do not address the stability of the continuity at $\mathcal{O}$ of a solution $f$ of (1)-(2) but we note that the procedure in [Shi and Finn 2004], as stated, would not establish the discontinuity at $\mathcal{O}$ of $f$ for arbitrarily small perturbations of the domain (in the asymptotic sense of Shi and Finn) when $\gamma_{1}=\gamma_{2}>\alpha-\frac{\pi}{2}$.

\section{Some lemmas}

Lemma 3. Let $\Omega$ be a bounded open domain in $\mathbb{R}^{2}$ with Lipschitz boundary and let $\Gamma$ be an open subset of $\partial \Omega$ which is a $C^{2, \beta}$ curve for some $\beta \in(0,1)$. Let 
$\gamma \in L^{\infty}(\partial \Omega)$ satisfy $\delta \leq \gamma \leq \pi-\delta$ a.e. on $\partial \Omega$ for some $\delta>0$ and $\gamma \in C^{1, \beta}(\Gamma)$. Suppose there exists $f \in C^{2}(\Omega) \cap L^{\infty}(\Omega)$ which satisfies

$$
\operatorname{div}(T u)=\kappa u \quad \text { in } \Omega
$$

and

$$
T u \cdot v=\cos \gamma \quad \text { on } \Gamma \text {. }
$$

Then $f \in C^{2, \beta}(\Omega \cup \Gamma)$.

See [Finn 1986, p. 210, Note 5], or [Finn 1988], or the introduction of [Korevaar and Simon 1996], which references [Simon and Spruck 1976; Taylor 1977].

The next result uses the notation of [Korevaar and Simon 1996, Theorem 2]; in particular,

$$
\frac{(\nabla g(x),-1)}{\sqrt{1+|\nabla g(x)|^{2}}}
$$

denotes the continuous extension of the (downward) unit normal to the graph of $g$ when considered as a function on this graph.

Lemma 4. Let $\Omega$ be a bounded open domain in $\mathbb{R}^{2}$ with Lipschitz boundary and let $\Gamma$ be an open subset of $\partial \Omega$ which is a $C^{3}$ curve. Let $\phi \in L^{\infty}(\partial \Omega)$ be in $C^{1, \beta}(\Gamma)$ for some $\beta \in(0,1)$. Suppose $g \in C^{2}(\Omega) \cap L^{\infty}(\Omega)$ is the variational solution of

$$
\begin{array}{ll}
\operatorname{div}(T u)=\kappa u & \text { in } \Omega, \\
u=\phi & \text { on } \partial \Omega ;
\end{array}
$$

that is, $g$ minimizes $J(\cdot)$ over $\mathrm{BV}(\Omega)$, where

$$
J(u)=\int_{\Omega} \sqrt{1+|D u|^{2}}+\int_{\Omega} \int_{0}^{u} \kappa t d t d x+\int_{\partial \Omega}|u-\phi| d s, \quad u \in \mathrm{BV}(\Omega) .
$$

Set

$$
Q=\{(x, t) \in \Gamma \times \mathbb{R}: \min \{\phi(x), g(x)\} \leq t \leq \max \{\phi(x), g(x)\}\}
$$

and $Q_{0}=Q \backslash T$, where $T \subset \partial \Omega \times \mathbb{R}$ is the graph of $\phi$, and let $G$ be the graph of $g$ over $\Omega$. Then for each $x_{0} \in \Gamma$, there exists a $\delta>0$ such that $\left\{x \in \partial \Omega:\left|x-x_{0}\right| \leq \delta\right\} \subset \Gamma$ and the following conclusions hold:

(a) $\Pi=\left\{(x, t) \in Q \cup G:\left|x-x_{0}\right| \leq \delta\right\}$ is a $C^{1, \sigma}$ manifold with boundary whose boundary is the union of $\left\{(x, \phi(x)) \in T: x \in \Gamma,\left|x-x_{0}\right| \leq \delta\right\}$ and $\{(x, g(x))$ : $\left.x \in \Omega,\left|x-x_{0}\right|=\delta\right\}$ for some $\sigma \in(0,1)$. 
(b) The (downward) unit normal $\vec{N}$ to $\Pi$ is a continuous function and

$$
\vec{N}(x, t)= \begin{cases}\frac{(\nabla g(x),-1)}{\sqrt{1+|\nabla g(x)|^{2}}} & \text { if } x \in \Omega \cup \Gamma \text { and } t=g(x), \\ (v(x), 0) & \text { if }(x, t) \in Q \text { and } g(x) \leq t<\phi(x), \\ (-v(x), 0) & \text { if }(x, t) \in Q \text { and } \phi(x)<t \leq g(x),\end{cases}
$$

where $v$ denotes the outward unit normal to $\partial \Omega$.

Proof. Let $A=\left\{x \in \Gamma: g^{*}(x)=\phi(x)\right\}, B=\left\{x \in \Gamma: g^{*}(x) \neq \phi(x)\right\}$, and $A_{0}$ be the interior (in $\Gamma$ ) of $A$, where $g^{*}$ is the trace of $g$ on $\partial \Omega$; let us define $g^{*}(x)$ to be $\phi(x)$ if $x \in \Gamma$ and $g^{*}(x)$ is not otherwise defined. Using the arguments in [Elcrat and Lancaster 1986], we see that if $x_{0} \in A_{0}$, then there exists a $\delta>0$ such that $\left\{x \in \partial \Omega:\left|x-x_{0}\right| \leq \delta\right\} \subset A_{0}$ and (a) and (b) hold.

Suppose $x_{0} \in B$ such that $g^{*}\left(x_{0}\right)=z_{0}<\phi\left(x_{0}\right)$ and so $\left(x_{0}, z_{0}\right)$ is an interior point of $\mathcal{Q} \cup G$. Standard results on the regularity of solutions of obstacle problems at interior points imply $g$ is continuous on $(\bar{\Omega} \times \mathbb{R}) \cap U$, where $U$ is a neighborhood in $\mathbb{R}^{3}$ of $\left(x_{0}, z_{0}\right)$, and, considered as a parametric surface, $(\mathcal{Q} \cup G) \cap U$ is a $C^{1, \alpha}$ surface for some $\alpha \in(0,1)$. (For example, this follows from [Simon and Spruck 1976] or [Taylor 1977], since the contact angle is zero at these interior points. Another argument follows from [Hildebrandt 1973]; by "blowing up" or dilating $\mathbb{R}^{3}$ about $\left(x_{0}, z_{0}\right)$, we may assume the function $f: E \times \mathbb{R}^{3} \times \mathbb{R}^{3} \times \mathbb{R}^{3} \rightarrow \mathbb{R}$ given by $f(w, X, p, q)=|p|^{2}+|q|^{2}+\frac{1}{2} \kappa\left(X_{3}-z_{0}\right)\left(\left(X-\left(x_{0}, z_{0}\right)\right) \cdot(p \times q)\right)$ satisfies conditions $\mathrm{A}$ and $\mathrm{B}$ of that paper in a neighborhood $U \times I$ of $\left(x_{0}, z_{0}\right)$, where $I$ is an open interval containing $z_{0}$, and so, for smooth Dirichlet data $\psi$ slightly larger than $z_{0}$ near $x_{0}$ and equal to $g$ on $\Omega \cap \partial U$, a theorem in [Hildebrandt 1973] shows there is a parametric minimizer of $\int_{E} f(w, z, \nabla z) d u d v$ that is smooth in the interior of its domain $E=\left\{(u, v): u^{2}+v^{2}<1\right\}$ and, from [Miranda 1964] (or [Finn 1986, p. 16, Note 10]), we see that this parametric solution is a graph $z=h(x, y)$. Since $g \leq h$ on $\partial U$ by the choice of $\psi$ and $h \leq g$ on $\partial U$ since $\psi<\phi$ on $U \cap \partial \Omega$, we see that $g=h$ in $U$. In particular, $g$ is continuous at each point of $\Gamma \cap B$ and the points of $A \backslash A_{0}$ are isolated.) An application of [Bourni 2011] shows that (a) and (b) hold; that is, we may choose a domain $\mathcal{V} \subset \Omega$ such that $\partial \mathcal{V}$ is a $C^{1, \alpha}$ curve in $\mathbb{R}^{2}, x_{0} \in \partial \mathcal{V} \subset \Gamma \cup \Omega, \partial \Omega \cap \overline{(\Omega \cap \partial \mathcal{V})}=\left\{x^{(j)}: j=1,2\right\}$ with $x^{(j)} \in B(j=1,2)$, the closure in $\mathbb{R}^{3}$ of $\{(x, g(x)) \in \Omega \times \mathbb{R}: x \in \partial \mathcal{V}\}$ is a $C^{1, \alpha}$ curve in $\mathbb{R}^{3}$ which meets $\Gamma \times \mathbb{R}$ tangentially at $\left(x^{(j)}, g^{*}\left(x^{(j)}\right)\right), j=1,2$, and we can find a function $\psi: \partial \mathcal{V} \rightarrow \mathbb{R}$ whose graph is a $C^{1, \alpha}$ curve in $\mathbb{R}^{3}$ such that $g^{*} \leq \psi \leq \phi$ on $\partial \Omega \cap \partial \mathcal{V}$ (see Figure 1(a)) and apply the conclusion of [Bourni 2011] to see that (a) and (b) hold in a neighborhood of $x_{0}$. If $x_{0} \in B$ such that $g^{*}\left(x_{0}\right)=z_{0}>\phi\left(x_{0}\right)$, apply the argument above to $-g$ (with $-\phi$ as Dirichlet data). 


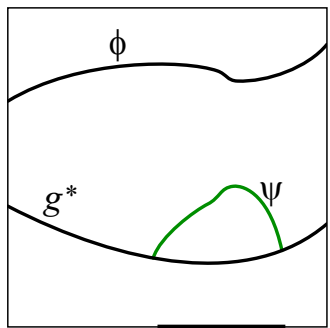

(a) $\partial v \cap \partial \Omega$

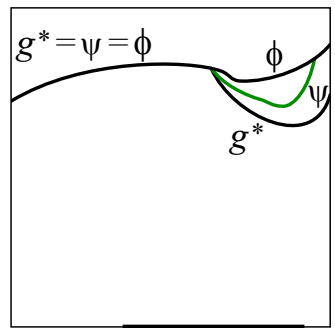

(b) $\partial v \cap \partial \Omega$

Figure 1. The traces of $g, \psi$ and $\phi$.

Suppose $x_{0} \in A \backslash A_{0}$. Notice, from the arguments above, that $A_{0}$ and $B$ are open. There exist a domain $\mathcal{V} \subset \Omega$ and a function $\psi: \partial \mathcal{V} \rightarrow \mathbb{R}$ as above such that $x_{0} \in \partial \mathcal{V} \subset \Gamma \cup \Omega$ and $\partial \Omega \cap \overline{(\Omega \cap \partial \mathcal{V})}=\left\{x^{(j)}: j=1,2\right\}$ with $x^{(j)} \in A_{0} \cup B$ $(j=1,2)$; we argue as above (see, for example, Figure 1(b)).

Lemma 5. Let $\Omega$ be a bounded open domain in $\mathbb{R}^{2}$ with Lipschitz boundary and let $\Gamma$ be an open subset of $\partial \Omega$ which is a $C^{4}$ curve or a finite disjoint union of $C^{4}$ curves. Let $\gamma \in L^{\infty}(\partial \Omega)$ satisfy $\delta \leq \gamma \leq \pi-\delta$ a.e. on $\partial \Omega$ for some $\delta>0$ and $\gamma \in C^{1, \beta}(\Gamma)$ for some $\beta \in(0,1)$. Suppose there exists $f \in C^{2}(\Omega) \cap L^{\infty}(\Omega)$ which satisfies (4) and (5). Let $\epsilon>0$. Define $g=g_{\epsilon} \in \mathrm{BV}(\Omega)$ to be the minimizer over $\mathrm{BV}(\Omega)$ of $J_{\epsilon}(\cdot)$, where

$$
J_{\epsilon}(u)=\int_{\Omega} \sqrt{1+|D u|^{2}}+\int_{\Omega} \int_{0}^{u} k t d t d x+\int_{\partial \Omega}|u-(f+\epsilon)| d s
$$

for $u \in \mathrm{BV}(\Omega)$. We have:

(i) $g \in C^{2}(\Omega)$ and satisfies (4).

(ii) The unit normal $\vec{N}$ to $\Pi$ is in $C^{0, \beta}(\Omega \cup E)$ for each compact subset $E$ of $\Gamma$ and hence the contact angle

$$
\gamma_{g} \stackrel{\text { def }}{=} \arccos (T g \cdot v) \in[0, \pi]
$$

is well defined and continuous on $\Gamma$, where $v$ denotes the outward unit normal to $\partial \Omega$. In particular, $\gamma_{g}=0$ on $\{x \in \Gamma: g(x)<f(x)+\epsilon\}$.

(iii) Suppose there is a finite set $A=\left\{x_{1}, \ldots, x_{m}\right\} \subset \partial \Omega$ such that $\Gamma=\partial \Omega \backslash A$. Then $f \leq g \leq f+\epsilon$ in $\Omega$.

(iv) Suppose there is a finite set $A=\left\{x_{1}, \ldots, x_{m}\right\} \subset \partial \Omega$ such that $\Gamma=\partial \Omega \backslash A$. Then $\gamma_{g}<\gamma$ on $\Gamma$.

Proof. (i) The existence of $g$ follows from Theorem 5 of [Gerhardt 1974], or Theorem 2.1 of [Giusti 1976]. The interior regularity of $g$ follows from Theorem 3.1 
of [Giusti 1976] (see also [Gerhardt 1974, p. 174; Williams 1978, Theorem 3]). The fact that $g$ satisfies (4) is standard (e.g., [Gerhardt 1974, p. 174]).

(ii) The boundary regularity of $g$ follows from Lemma 4. On $\{x \in \Gamma: g(x)<$ $f(x)+\epsilon\}$, we have $\vec{N}(x, g(x))=(v(x), 0), T g(x)=v(x)$, and so

$$
\gamma_{g}(x)=\arccos (v(x) \cdot v(x))=\arccos (1)=0 .
$$

(iii) Notice that $f, g \in C^{2}(\Omega) \cap C^{0}(\Omega \cup \Gamma)$. Set $M=\{x \in \Omega: f(x)>g(x)\}$. On $\partial M \cap \Gamma, g<f+\epsilon$ and so by (ii) and Lemma 4, with $\Pi=Q \cup G$, where $Q=$ $\{(x, z):(x, z) \in E, g(x) \leq z<f(x)\} \in M \times \mathbb{R}$, implies that sup $\cos \gamma_{g}=T g \cdot v=1$; hence $\gamma_{g}=0$ on $\partial M \cap \Gamma$. Thus $f=g$ on $\Omega \cap \partial M$ and $\gamma_{g}=0$ almost everywhere on $\partial \Omega \cap \partial M$ and so the general comparison principle (e.g., [Finn 1986, Theorem 5.1]) implies $f \leq g$ in $M$; hence $M=\varnothing$.

Now let $\tau>0$ and set $N=\{x \in \Omega: g(x)>f(x)+\epsilon+\tau\}$. Then $g=f+\epsilon+\tau$ on $\Omega \cap \partial N$ and $g>f+\epsilon$ on $\partial N \cap \Gamma$ and so Lemma 4 implies $\gamma_{g}=\pi$ almost everywhere on $\partial \Omega \cap \partial N$. The general comparison principle then implies $g \leq f+\epsilon+\tau$ and so $N=\varnothing$. Therefore $g \leq f+\epsilon+\tau$ in $\Omega$ for each $\tau>0$ and so $g \leq f+\epsilon$ in $\Omega$.

(iv) Suppose first $x \in \Gamma$ and there is a sequence $\left\{y_{j}\right\}$ in $\Gamma$ such that $x=\lim _{j \rightarrow \infty} y_{j}$ and $g\left(y_{j}\right)<f\left(y_{j}\right)+\epsilon$ for each $j$. Then (ii) implies $\gamma_{g}\left(y_{j}\right)=0$ for each $j$ and so $\gamma_{g}(x)=0$. Since $\gamma \in(0, \pi)$, we see that $\gamma_{g}(x)=0<\gamma(x)$.

Suppose next that $x \in \Gamma$ and $g \geq f+\epsilon$ in $\mathcal{P} \cap \Gamma$, where $\mathcal{P}$ is a neighborhood of $x$ in $\mathbb{R}^{2}$. From (iii), we see that $g=f+\epsilon$ in $\mathcal{P} \cap \Gamma$. If $\gamma_{g}(x)>\gamma(x)$, then $g(x-t v(x))>f(x-t v(x))+\epsilon$ for $t>0$ small and this contradicts (iii). (Recall that $\nu(x)$ is the exterior unit normal to $\partial \Omega$ at $x$.) Thus $\gamma_{g} \leq \gamma$ on $\Gamma$.

Finally, suppose $x \in \Gamma, \gamma_{g}(x)=\gamma(x)$ and $g=f+\epsilon$ in $\mathcal{P} \cap \partial \Omega$, where $\mathcal{P}$ is a neighborhood of $x$ in $\mathbb{R}^{2}$; notice that [Heinz 1970] and Lemma 3 imply $g \in$ $C^{2, \beta}(\mathcal{P} \cap \bar{\Omega})$. Since $g \leq f+\epsilon$ in $\Omega$ and $\gamma_{g}(x)=\gamma(x)$, the tangent plane $\Pi_{g}$ to $z=g$ at $(x, g(x))$ and the tangent plane $\Pi$ to $z=f+\epsilon$ at $(x, g(x))=(x, f(x)+\epsilon)$ must coincide. Now the mean curvature $H_{g}$ of $z=g$ at $(x, g(x))$ is $\frac{1}{2} \kappa g(x)$ and the mean curvature $H_{f}$ of $z=f+\epsilon$ at $(x, g(x))$ is $\frac{1}{2} \kappa f(x)=\frac{1}{2}(\kappa g(x)-\kappa \epsilon)$. Since $g=f+\epsilon$ in $\mathcal{P} \cap \Gamma$, the (signed) curvature of the curve $z=f(x-t v(x))+\epsilon$ must be strictly less than the (signed) curvature of the curve $z=g(x-t v(x))$ for $t>0$ small and so $g(x-t v(x))>f(x-t v(x))+\epsilon$ for $t>0$ small, contradicting (iii).

\section{Stability of central fans}

We will begin by establishing the stability of the central fans with respect to "onesided" perturbations of $\gamma$. (See Figures 2 and 3.)

Theorem 6. Let $\Omega$ be an open, connected, bounded Lipschitz domain such that $\mathcal{O}=(0,0) \in \partial \Omega, \Omega$ has a corner at $\mathcal{O}$ with opening angle $2 \alpha>\pi$ and there is a finite set $A=\left\{P_{1}, \ldots, P_{m}\right\} \subset \partial \Omega$ with $m \geq 1$ and $P_{1}=\mathcal{O}$ such that $\partial \Omega \backslash A$ is 


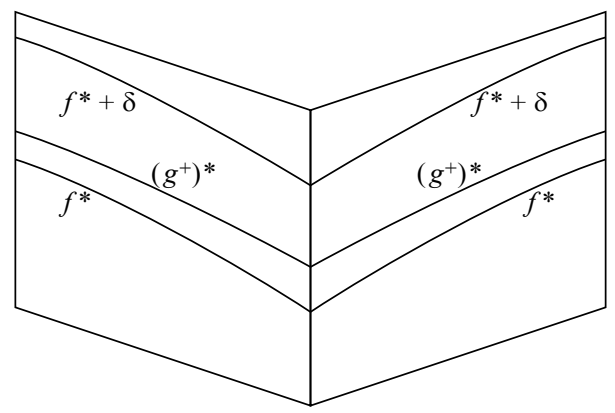

Figure 2. The traces of $f, g^{+}$and $f+\delta$.

$a C^{4}$ curve (if $m=1$ ) or a finite disjoint union of $C^{4}$ curves (if $m>1$ ). Suppose $\gamma: \partial \Omega \backslash \mathcal{O} \rightarrow(0, \pi)$ is a $C^{1, \beta}$ map for which the limits

$$
\lim _{\partial^{+} \Omega \ni(x, y) \rightarrow \mathcal{O}} \gamma(x, y)=\gamma_{1}, \quad \lim _{\partial^{-} \Omega \ni(x, y) \rightarrow \mathcal{O}} \gamma(x, y)=\gamma_{2}
$$

exist with $\gamma_{i} \in(0, \pi), i=1,2$, and $f \in C^{2}(\Omega) \cap C^{1, \beta}(\bar{\Omega} \backslash\{\mathcal{O}\})$ satisfies

$$
\begin{array}{ll}
\operatorname{div}(T f)=\kappa f & \text { in } \Omega, \\
T f \cdot v=\cos \gamma & \text { on } \partial \Omega \backslash\{\mathcal{O}\}
\end{array}
$$

such that $f$ is discontinuous at $\mathcal{O}$ and the radial limits $R f(\cdot)$ of $f$ at $\mathcal{O}$ have a central fan.

There exists a $\delta>0$ such that if $g^{+} \in \mathrm{BV}(\Omega) \cap C^{2}(\Omega)$ is the variational solution of the Dirichlet problem

$$
\begin{array}{ll}
\operatorname{div}(T g)=\kappa g & \text { in } \Omega, \\
g=f+\delta & \text { on } \partial \Omega \backslash A,
\end{array}
$$

and if $\omega^{+} \stackrel{\text { def }}{=} \arccos \left(T g^{+} \cdot v\right)$ on $\partial \Omega \backslash A$, then for any function $\sigma \in L^{\infty}(\partial \Omega)$ satisfying

$$
\omega^{+} \leq \sigma \leq \gamma \quad \text { a.e. on } \partial \Omega
$$

and $\delta_{1} \leq \sigma \leq \pi-\delta_{1}$ for some $\delta_{1}>0$, the variational solution $h \in \mathrm{BV}(\Omega) \cap C^{2}(\Omega)$ of the capillary problem

$$
\operatorname{div}(T h)=\kappa h \text { in } \Omega, \quad T h \cdot v=\cos \sigma \text { on } \partial \Omega \backslash A
$$

is discontinuous at $\mathcal{O}$, the radial limits $R h(\cdot)$ of $h$ at $\mathcal{O}$ have a central fan and they have the same type of behavior (i.e., case (DI) or (ID)) as $R f(\cdot)$.

Proof. Suppose first that $R f$ behaves as in case (DI) and so

$$
R f(0)<\min \{R f(\alpha), R f(-\alpha)\} .
$$




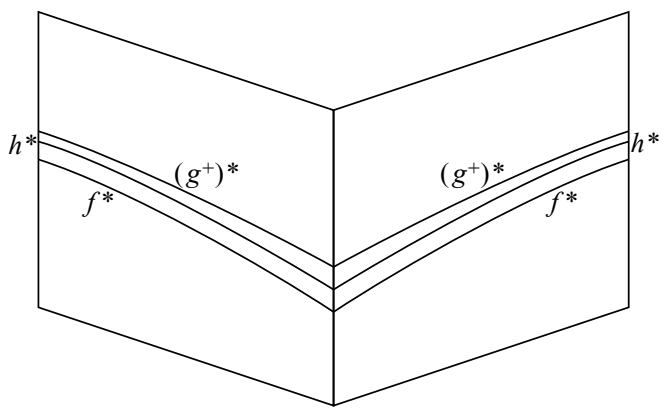

Figure 3. The traces of $f, h$ and $g^{+}$.

Let $\delta<\min \{R f(\alpha)-R f(0), R f(-\alpha)-R f(0)\}$ and let $g^{+}$be the variational solution of the Dirichlet problem (7)-(8) for this choice of $\delta$ (see Figure 2). From Lemma 5(iv), we see that $\omega^{+}<\gamma$ on $\partial \Omega \backslash A$ and therefore there exist $\sigma \in L^{\infty}(\partial \Omega$ ) which satisfy (9); let us select $\sigma$ and $h$ as in the theorem (see Figure 3). From Lemma 5(iii) and the general comparison principle, we see that $f \leq h \leq g^{+} \leq f+\delta$ in $\Omega$ and hence

$$
R f(\theta) \leq R h(\theta) \leq R g^{+}(\theta) \leq R f(\theta)+\delta \quad \text { for } \theta \in[-\alpha, \alpha] ;
$$

thus

$$
R h(\alpha)-R h(0) \geq R f(\alpha)-(R f(0)+\delta)=R f(\alpha)-R f(0)-\delta>0
$$

and

$$
R h(-\alpha)-R h(0) \geq R f(-\alpha)-(R f(0)+\delta)=R f(-\alpha)-R f(0)-\delta>0 .
$$

Now we know that the radial limits of $h$ at $\mathcal{O}$ exist and behave as in [Lancaster and Siegel 1996] (i.e., one of case (I), (D), (ID) or (DI) must hold; if, for example, case (I) held, one would have $R f(-\alpha)<R f(0)<R f(\alpha))$. The calculations above show that $R f(-\alpha)>R f(0)$ and $R f(\alpha)>R f(0)$ and therefore $R h(\cdot)$ must behave as in case (DI); hence $R h(\cdot)$ has a central fan (see Figure 4$)$.

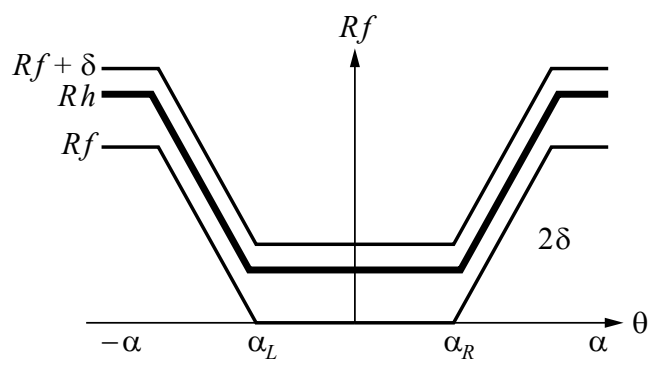

Figure 4. The radial limits of $f, h$ and $f+\delta$. 
Suppose next that $R f$ behaves as in case (ID) and so

$$
R f(0)>\min \{R f(\alpha), R f(-\alpha)\} .
$$

If we let $\delta<\min \{R f(0)-R f(\alpha), R f(0)-R f(-\alpha)\}$, we may repeat the same argument as above and obtain $R h(0)>R h(-\alpha)$ and $R h(0)>R h(\alpha)$; hence $R h(\cdot)$ must behave as in case (ID) and therefore has a central fan.

Remark 7. The corresponding theorem with (8) replaced by

$$
g^{-}=f-\delta \quad \text { on } \partial \Omega \backslash A
$$

and with $\omega^{-} \stackrel{\text { def }}{=} \arccos \left(T g^{-} \cdot v\right)$ on $\partial \Omega \backslash A, \omega^{-} \geq \sigma \geq \gamma$ on $\partial \Omega \backslash A, \delta_{1} \leq \sigma \leq \pi-\delta_{1}$ for some $\delta_{1}>0$ and $h$ a solution of (10) yields $f-\delta \leq g^{-} \leq h \leq f$ in $\Omega$ and

$$
R f(\theta) \leq R h(\theta) \leq R f(\theta)+\delta \quad \text { for } \theta \in[-\alpha, \alpha] ;
$$

hence $h$ is discontinuous at $\mathcal{O}$ and the radial limits $R h(\cdot)$ of $h$ at $\mathcal{O}$ have the same type of behavior (i.e., case (DI) or (ID)) as $R f(\cdot)$.

Proof of Theorem 1. Suppose $R f$ behaves as in case (DI) or case (ID) and define $\delta=\frac{1}{2} \min \{|R f(\alpha)-R f(0)|,|R f(-\alpha)-R f(0)|\}$. Combining the arguments in Theorem 6 and Remark 7, we obtain

$$
R f(\alpha)-R f(0)-\delta \leq R h(\alpha)-R h(0) \leq R f(\alpha)-R f(0)+\delta
$$

and

$$
R f(-\alpha)-R f(0)-\delta \leq R h(\alpha)-R h(0) \leq R f(-\alpha)-R f(0)+\delta .
$$

If $R f$ behaves as in case (DI), we have $0<R h(\alpha)-R h(0)$ and $0<R h(-\alpha)-R h(0)$ and therefore $R h$ behaves as in case (DI). If $R f$ behaves as in case (ID), we have $R h(\alpha)-R h(0)<0$ and $R h(-\alpha)-R h(0)<0$ and therefore $R h$ behaves as in case (ID).

Proof of Corollary 2. Since $\gamma_{0}<\alpha-\frac{\pi}{2}$, we see that $|2 \gamma-\pi|>2 \pi-2 \alpha$. It follows from [Lancaster 2012] that $f$ is discontinuous at $\mathcal{O}$. Since $f(x, y)=f(x,-y)$ for each $(x, y) \in \Omega$, the radial limits of $f$ cannot behave as in cases (I) or (D) of Theorem 1 of [Lancaster and Siegel 1996] and therefore they have a central fan. That case (DI) holds for $R f(\cdot)$ follows from [Lancaster 2012] or directly from the fact that $\left(\pi-\gamma_{0}\right)+\left(\pi-\gamma_{0}\right)+\pi>4 \pi-2 \alpha>2 \alpha$ means case (ID) cannot hold. The claim follows from Theorem 1 .

Remark 8. It should be emphasized that the conclusion of Theorem 1 is not that "there exists a $\delta>0$ such that if $\sigma: \partial \Omega \rightarrow[0, \pi]$ satisfies $\gamma-\delta \leq \sigma \leq \gamma+\delta$ a.e. on $\partial \Omega$, then the radial limit function $R h$ of the solution $h \in C^{2}(\Omega)$ of (1)-(2) with $\gamma$ replaced by $\sigma$ in (2) has the same type of behavior (i.e., case (ID) or case (DI) 
holds) as does $R f$ ". The validity of such a conclusion is an interesting question which might spur further investigation.

\section{Acknowledgements}

The author wishes to thank Professor K. Lancaster for his valuable comments and suggestions throughout this research, and also to thank the editor and referee for their patience and to acknowledge the contributions of the referee, who provided many helpful comments and Figure 4.

\section{References}

[Bourni 2011] T. Bourni, " $C^{1, \alpha}$ theory for the prescribed mean curvature equation with Dirichlet data”, J. Geom. Anal. 21:4 (2011), 982-1035. MR 2836589 Zbl 1232.53009

[Elcrat and Lancaster 1986] A. R. Elcrat and K. E. Lancaster, "Boundary behavior of a nonparametric surface of prescribed mean curvature near a reentrant corner", Trans. Amer. Math. Soc. 297:2 (1986), 645-650. MR 87h:35098 Zbl 0602.35042

[Finn 1986] R. Finn, Equilibrium capillary surfaces, Grundlehren der Mathematischen Wissenschaften 284, Springer, New York, 1986. MR 88f:49001 Zbl 0583.35002

[Finn 1988] R. Finn, "Moon surfaces, and boundary behaviour of capillary surfaces for perfect wetting and nonwetting", Proc. London Math. Soc. (3) 57:3 (1988), 542-576. MR 89m:49076 Zbl 0668.76019

[Gerhardt 1974] C. Gerhardt, "Existence, regularity, and boundary behaviour of generalized surfaces of prescribed mean curvature", Math. Z. 139 (1974), 173-198. MR 55 \#10846 Zbl 0316.49005

[Giusti 1976] E. Giusti, "Boundary value problems for non-parametric surfaces of prescribed mean curvature”, Ann. Scuola Norm. Sup. Pisa Cl. Sci. (4) 3:3 (1976), 501-548. MR 58 \#2572 Zbl 0344.35036

[Heinz 1970] E. Heinz, “Über das Randverhalten quasilinearer elliptischer Systeme mit isothermen Parametern”, Math. Z. 113 (1970), 99-105. MR 41 \#7288 Zbl 0176.41004

[Hildebrandt 1973] S. Hildebrandt, "Interior $C^{1+\alpha}$-regularity of solutions of two-dimensional variational problems with obstacles”, Math. Z. 131 (1973), 233-240. MR 51 \#1568 Zbl 0275.49007

[Korevaar and Simon 1996] N. Korevaar and L. Simon, "Equations of mean curvature type with contact angle boundary conditions", pp. 175-201 in Geometric analysis and the calculus of variations, edited by J. Jost, International Press, Cambridge, MA, 1996. MR 98e:35071 Zbl 0932.35091

[Lancaster 1985] K. E. Lancaster, "Boundary behavior of a nonparametric minimal surface in $\mathbb{R}^{3}$ at a non-convex point”, Analysis 5:1-2 (1985), 61-69. Corrigendum in 6:4 (1986), 413. MR 86m:49053 Zbl 0601.35035

[Lancaster 2010] K. E. Lancaster, "A proof of the Concus-Finn conjecture", Pacific J. Math. 247:1 (2010), 75-108. MR 2011m:53011 Zbl 1256.53009

[Lancaster 2012] K. E. Lancaster, "Remarks on the behavior of nonparametric capillary surfaces at corners”, Pacific J. Math. 258:2 (2012), 369-392. MR 2981959 Zbl 1253.35069

[Lancaster and Siegel 1996] K. E. Lancaster and D. Siegel, "Existence and behavior of the radial limits of a bounded capillary surface at a corner", Pacific J. Math. 176:1 (1996), 165-194. MR 98g:58030a Zbl 0866.76018 
[Miranda 1964] M. Miranda, "Superfici Cartesiane generalizzate ed insiemi di perimetro localmente finito sui prodotti Cartesiani”, Ann. Scuola Norm. Sup. Pisa (3) 18 (1964), 515-542. MR 30 \#4906 Zbl 0152.24402

[Shi and Finn 2004] D. Shi and R. Finn, "On a theorem of Lancaster and Siegel", Pacific J. Math. 213:1 (2004), 111-119. MR 2004m:76038 Zbl 1156.76361

[Simon and Spruck 1976] L. Simon and J. Spruck, "Existence and regularity of a capillary surface with prescribed contact angle”, Arch. Rational Mech. Anal. 61:1 (1976), 19-34. MR 58 \#7339 Zbl 0361.35014

[Taylor 1977] J. E. Taylor, "Boundary regularity for solutions to various capillarity and free boundary problems”, Comm. Partial Differential Equ. 2:4 (1977), 323-357. MR 58 \#7336 Zbl 0357.35010

[Williams 1978] G. H. Williams, "Surfaces of prescribed mean curvature with inequalities on the boundary”, Math. Z. 164:1 (1978), 31-51. MR 80d:53006 Zbl 0378.49029

Received August 30, 2013. Revised August 11, 2015.

AMMAR KHANFER

DEPARTMENT OF MATHEMATICS AND STATISTICS

AL-IMAm MOHAMmad IBN SAUd ISLAMIC UNIVERSITY

P.O. BOX 90950

AL-RIYADH 11623

SAUDI ARABIA

a.khanfer@yahoo.com 


\title{
PACIFIC JOURNAL OF MATHEMATICS
}

\author{
msp.org/pjm
}

Founded in 1951 by E. F. Beckenbach (1906-1982) and F. Wolf (1904-1989)

\section{EDITORS}

Don Blasius (Managing Editor)

Department of Mathematics

University of California

Los Angeles, CA 90095-1555

blasius@math.ucla.edu

\author{
Paul Balmer \\ Department of Mathematics \\ University of California \\ Los Angeles, CA 90095-1555 \\ balmer@math.ucla.edu \\ Robert Finn \\ Department of Mathematics \\ Stanford University \\ Stanford, CA 94305-2125 \\ finn@math.stanford.edu \\ Sorin Popa \\ Department of Mathematics \\ University of California \\ Los Angeles, CA 90095-1555 \\ popa@math.ucla.edu
}

\author{
Vyjayanthi Chari \\ Department of Mathematics \\ University of California \\ Riverside, CA 92521-0135 \\ chari@math.ucr.edu \\ Kefeng Liu \\ Department of Mathematics \\ University of California \\ Los Angeles, CA 90095-1555 \\ liu@math.ucla.edu \\ Jie Qing \\ Department of Mathematics \\ University of California \\ Santa Cruz, CA 95064 \\ qing@ cats.ucsc.edu
}

\section{PRODUCTION}

Silvio Levy, Scientific Editor, production@msp.org

\section{SUPPORTING INSTITUTIONS}

ACADEMIA SINICA, TAIPEI

CALIFORNIA INST. OF TECHNOLOGY

INST. DE MATEMÁTICA PURA E APLICADA

KEIO UNIVERSITY

MATH. SCIENCES RESEARCH INSTITUTE

NEW MEXICO STATE UNIV.

OREGON STATE UNIV.

\author{
STANFORD UNIVERSITY \\ UNIV. OF BRITISH COLUMBIA \\ UNIV. OF CALIFORNIA, BERKELEY \\ UNIV. OF CALIFORNIA, DAVIS \\ UNIV. OF CALIFORNIA, LOS ANGELES \\ UNIV. OF CALIFORNIA, RIVERSIDE \\ UNIV. OF CALIFORNIA, SAN DIEGO \\ UNIV. OF CALIF., SANTA BARBARA
}

\author{
Daryl Cooper \\ Department of Mathematics \\ University of California \\ Santa Barbara, CA 93106-3080 \\ cooper@math.ucsb.edu \\ Jiang-Hua Lu \\ Department of Mathematics \\ The University of Hong Kong \\ Pokfulam Rd., Hong Kong \\ jhlu@maths.hku.hk \\ Paul Yang \\ Department of Mathematics \\ Princeton University \\ Princeton NJ 08544-1000 \\ yang@math.princeton.edu
}

These supporting institutions contribute to the cost of publication of this Journal, but they are not owners or publishers and have no responsibility for its contents or policies.

See inside back cover or msp.org/pjm for submission instructions.

The subscription price for 2016 is US $\$ 440 /$ year for the electronic version, and $\$ 600 /$ year for print and electronic.

Subscriptions, requests for back issues and changes of subscribers address should be sent to Pacific Journal of Mathematics, P.O. Box 4163, Berkeley, CA 94704-0163, U.S.A. The Pacific Journal of Mathematics is indexed by Mathematical Reviews, Zentralblatt MATH, PASCAL CNRS Index, Referativnyi Zhurnal, Current Mathematical Publications and Web of Knowledge (Science Citation Index).

The Pacific Journal of Mathematics (ISSN 0030-8730) at the University of California, c/o Department of Mathematics, 798 Evans Hall \#3840, Berkeley, CA 94720-3840, is published twelve times a year. Periodical rate postage paid at Berkeley, CA 94704, and additional mailing offices. POSTMASTER: send address changes to Pacific Journal of Mathematics, P.O. Box 4163, Berkeley, CA 94704-0163.

PJM peer review and production are managed by EditFLOW ${ }^{\circledR}$ from Mathematical Sciences Publishers.

\section{PUBLISHED BY}

\section{mathematical sciences publishers \\ nonprofit scientific publishing}

http://msp.org/

(C) 2016 Mathematical Sciences Publishers 


\title{
PACIFIC JOURNAL OF MATHEMATICS
}

\author{
Volume $281 \quad$ No. $2 \quad$ April 2016
}

The Eisenstein elements of modular symbols for level product of two 257 distinct odd primes

DEBARGHA BANERJEE and SRILAKSHMI KRISHNAMOORTHY

Primitively generated Hall algebras

ARKADY BERENSTEIN and JACOB GREENSTEIN

Generalized splines on arbitrary graphs

Simcha Gilbert, JULiAnNA TyMOCZKO and SHIRA ViEL

Good traces for not necessarily simple dimension groups

DAVID HANDELMAN

On Fourier coefficients of certain residual representations of symplectic groups

DIHUA JIANG and BAIYING LIU

On the existence of central fans of capillary surfaces

AMMAR KHANFER

Surfaces of prescribed mean curvature $H(x, y, z)$ with one-to-one central projection onto a plane

FRIEDRICH SAUVIGNY 